\title{
RS-34 \\ (Peacekeeper Post Boost Propulsion System) Orbital Debris Application Concept Study
}

\author{
Abstract \\ Elizabeth A. Esther, Christopher G. Burnside \\ NASA, George C. Marshall Space Flight Center, AL, 35812, United States
}

The Advanced Concepts Office (ACO) at the NASA Marshall Space Flight Center (MSFC) lead a study to evaluate the Rocketdyne produced RS-34 propulsion system as it applies to an orbital debris removal design reference mission. The existing RS-34 propulsion system is a remaining asset from the de-commissioned United States Air Force Peacekeeper ICBM program; specifically the pressure-fed storable bi-propellant Stage IV Post Boost Propulsion System.

MSFC gained experience with the RS-34 propulsion system on the successful Ares I-X flight test program flown in the Ares I-X Roll control system (RoCS). The heritage hardware proved extremely robust and reliable and sparked interest for further utilization on other potential in-space applications. Subsequently, MSFC is working closely with the USAF to obtain all the remaining RS-34 stages for re-use opportunities. Prior to pursuit of securing the hardware, MSFC commissioned the Advanced Concepts Office to understand the capability and potential applications for the RS-34 Phoenix stage as it benefits NASA, DoD, and commercial industry.

Originally designed, the RS-34 Phoenix provided in-space six-degrees-of freedom operational maneuvering to deploy multiple payloads at various orbital locations. The RS-34 Concept Study, preceded by a utilization study to understand how the unique capabilities of the RS-34 Phoenix and its application to six candidate missions, sought to further understand application for an orbital debris design reference mission as the orbital debris removal mission was found to closely mimic the heritage RS-34 mission.

The RS-34 Orbital Debris Application Concept Study sought to identify multiple configurations varying the degree of modification to trade for dry mass optimization and propellant load for overall capability and evaluation of several candidate missions. The results of the RS-34 Phoenix Utilization Study show that the system is technically sufficient to successfully support all of the missions analyzed.

The results and benefits of the RS-34 Orbital Debris Application Concept Study are presented in this paper. 